\title{
The effect of gadolinium doping on the structural, magnetic and photoluminescence properties of electrospun bismuth ferrite nanofibers
}

\author{
Geo George Philip ${ }^{\mathrm{a}}$, Anitha Senthamizhan ${ }^{\mathrm{b}, 1}$, Thirupathur Srinivasan Natarajan ${ }^{\mathrm{b}}$, \\ Gopalakrishnan Chandrasekaran ${ }^{\text {a }}$, Helen Annal Therese ${ }^{a, *}$ \\ ${ }^{a}$ Nanotechnology Research Centre, SRM University, Kattankulathur 603203, Tamil Nadu, India \\ ${ }^{\mathrm{b}}$ Department of Physics, Indian Institute of Technology Madras, Chennai 600036, Tamil Nadu, India
}

Received 6 March 2015; received in revised form 11 July 2015; accepted 20 July 2015

Available online 29 July 2015

\begin{abstract}
Gadolinium (Gd) doped Bismuth ferrite (BFO) nanofibers $\left(\mathrm{Bi}_{1-x} \mathrm{Gd}_{x} \mathrm{FeO}_{3}(x=0.0,0.05,0.10,0.15\right.$ and 0.20$)$ ) were synthesized via electrospinning. Scanning Electron Microscope (SEM) analysis showed that the diameter of the nanofibers ranged from 150 to $250 \mathrm{~nm}$. X-Ray Diffraction (XRD) analysis revealed a structural phase transition with varying ' $x$ ', the compositions with $x \leq 0.10$ have crystal structures with space group R3c, while the compositions with $x>0.10$ have crystal structures with space group Pnma. Vibrating Sample Magnetometer (VSM) analysis exhibited the weak ferromagnetic nature of the BFO nanofibers. However an increase in the saturated magnetic moment with increase in Gd dopant concentration was observed. The Photoluminescence (PL) spectra of the $\mathrm{Bi}_{1-x} \mathrm{Gd}_{x} \mathrm{FeO}_{3}$ nanofibers show enhanced Near Band Emission (NBE) intensity at $x=0.10$ due to the passivation of oxygen vacancies by Gd doping.
\end{abstract}

(c) 2015 Elsevier Ltd and Techna Group S.r.l. All rights reserved.

Keywords: Bismuth ferrite; Gadolinium doping; Nanostructured materials; Nanofabrication; Magnetic measurements; Luminescence

\section{Introduction}

Bismuth Ferrite (BFO) is the only single phase room temperature magnetoelectric multiferroic material [1]. It has a rhombohedral perovskite structure with a R3c space group [1]. It has been in the spotlight for its potential use in applications such as magnetic data recording [2], spintronics [3] and terahertz radiation [4]. In addition to its multiferroic properties, it has also garnered considerable attention for its optoelectronic properties. It has a bandgap varying from $2.5 \mathrm{eV}$ to $2.8 \mathrm{eV}$ [5-7]. It has been investigated as a potential candidate for optoelectronic applications such as photovoltaics [8-10], photonic and optoelectronic devices $[11,12]$. Studies have shown that the photoluminescence properties of BFO can even be manipulated through the use of electric fields

\footnotetext{
*Corresponding author. Tel.: +91 44 27417191(O), +91 8870828380(R); fax: +914427452343 .

E-mail address: helen.a@ktr.srmuniv.ac.in (H. Annal Therese).

${ }^{1}$ Present address: UNAM-National Nanotechnology Research Center, Bilkent University, Ankara 06800, Turkey.
}

[13]. Further, the optoelectronic properties of BFO have been found to be enhanced in one dimensional nanostructures such as nanofibers [11] and nanotubes [12] when compared to bulk samples, due to strong surface level interactions and large surface to volume ratio [14]. Electrospun nanofibers have added advantages over other one dimensional nanostructures such as high aspect ratios, large surface areas [15-17] and enhanced optical properties $[11,18]$. Studies on the effect of various processing conditions on the Near Band Emission (NBE) and Defect Level Emission (DLE) of BFO thin films [10] and nanowires [11] showed that the strength of the DLE band varied highly on the atmosphere such as $\mathrm{O}_{2}, \mathrm{~N}_{2}, \mathrm{H}_{2}$ or Air used during annealing. Both BFO nanofilms and wires showed strong DLE bands when annealed under $\mathrm{O}_{2}$, whereas nanowires annealed under $\mathrm{H}_{2}$ atmospheres exhibited weaker DLE bands. Recent studies on BFO nanotubes [12] synthesized via a sol-gel technique showed the presence of abundant oxygen vacancies, which affected the PL and photo absorption properties of the BFO nanotubes. Similarly reports on transition metal doping such as Nickel on the photoluminescence properties of BFO showed an increase in oxygen 
vacancies for higher levels of Nickel doping $[19,20]$. However, the effect of rare earth dopants such as Gadolinium $(\mathrm{Gd})$ on the photoluminescence properties of BFO system has not been investigated to the best of our knowledge. Doping of rare earth elements such as Gd into the BFO system has been shown to dramatically improve its magnetic and ferroelectric properties [21]. In this study, we investigate the effect of doping different concentrations of $\mathrm{Gd}$ into electrospun BFO nanofibers on their structural, magnetic and photoluminescence properties.

\section{Experimental}

\subsection{Materials and instruments}

Poly Vinyl Pyrrolidone (PVP) (Molecular weight -1.3 million) from Sigma Aldrich and Bismuth Nitrate pentahydrate $\left(\mathrm{Bi}\left(\mathrm{NO}_{3}\right)_{3} \cdot 5 \mathrm{H}_{2} \mathrm{O}(99.9 \%\right.$ purity)), Iron Nitrate nonahydrate $\left(\mathrm{Fe}\left(\mathrm{NO}_{3}\right)_{3} \cdot 9 \mathrm{H}_{2} \mathrm{O}(99.9 \%\right.$ purity)) and Gadolinium acetate $(\mathrm{Gd}$ $\left(\mathrm{CH}_{3} \mathrm{CO}_{2}\right)_{3} \cdot x \mathrm{H}_{2} \mathrm{O}(99.9 \%$ purity)) from Loba Chemie were used without any further processing. 2-methoxy ethanol, Glacial acetic acid and ethanol (99.9\% purity) were acquired from Rankem Chemicals and used as solvents. The nanofibers were synthesized in an electrospinning unit (ESPIN-NANO, PICO) and subsequently annealed in a muffle furnace. The morphology, crystallinity and the magnetic properties of the nanofibers were characterized by Field Emission Scanning Electron Microscope (FESEM) (FEG 200, FEI), X-Ray Diffractometer (XRD) (Xpert Pro, Panalytical), and Vibrating Sample Magnetometer (VSM) (Lakeshore VSM 7410) respectively. The Photoluminescence (PL) spectra of the nanofibers were studied using a Spectrofluorometer (Fluorolog - 4, Horiba Jobin Yvon). During PL measurements, the slit width at both the excitation and emission ends of the spectrofluorometer was kept at $5 \mathrm{~nm}$. The nanofibers $(\sim 100 \mathrm{mg})$ were packed to the brim of the solid sample holder (1933) to prevent any scattering during measurement.

\subsection{Synthesis of nanofibers}

Bismuth nitrate and Iron nitrate were dissolved in 2methoxy ethanol to produce a homogenous $\mathrm{BiFeO}_{3}$ precursor solution. Poly Vinyl Pyrrolidone (PVP) was dissolved in ethanol and mixed with precursor solution to form the electrospinning solution. Glacial acetic acid was added to maintain the $\mathrm{pH}$ of the sol to a value of 3.5 and to increase the conductivity of the electrospinning solution. The solution was subjected to a voltage of $9 \mathrm{kV}$, the distance between the collector and the syringe nozzle was kept at $18 \mathrm{~cm}$ and the flow rate was set as $0.5 \mathrm{ml} / \mathrm{h}$. The electrospun nanofibers were collected on a drum collector and then heated in a muffle furnace at a temperature of $500{ }^{\circ} \mathrm{C}$ for a period of $1 \mathrm{~h}$. The above synthesis procedure was repeated with addition of gadolinium acetate for the fabrication of $\mathrm{Gd}$ doped $\mathrm{BFO}$ nanofibers.

\section{Results and discussions}

\subsection{FESEM analyses}

The SEM images of as spun and annealed BFO nanofibers are shown in Fig. 1. The as spun composite fibers are seen to have a smooth morphology and have diameters ranging between $300 \mathrm{~nm}$ and $450 \mathrm{~nm}$. The annealed nanofibers have a grainy and rough morphology and have diameters ranging from $150 \mathrm{~nm}$ to $250 \mathrm{~nm}$. The $\mathrm{Gd}$ doped $\mathrm{BFO}$ nanofibers showed no change in morphology and diameter when compared with the undoped BFO nanofibers.

\subsection{XRD analyses}

The XRD patterns for $\mathrm{Gd}$ doped BFO nanofibers $\left(\mathrm{Bi}_{1-x} \mathrm{Gd}_{x-}\right.$ $\mathrm{FeO}_{3}(x=0.00,0.05,0.10,0.15$ and 0.20$\left.)\right)$ are shown in Fig. 2. Rietveld refinement analysis of all the XRD patterns shows that BFO nanofibers have a rhombohedral crystal structure in R3c space group arrangement, with lattice constant values of $a=5.5847 \AA$ and $c=13.8717 \AA$. As the quantity of dopant increases i.e at higher values of $x(x=0.15$ and $x=0.20)$, the formation of mixture of, rhombohedral and orthorhombic phases of $\mathrm{Bi}_{1-x} \mathrm{Gd}_{x} \mathrm{FeO}_{3}$ is observed. The XRD patterns were analyzed for the orthorhombic phases with a space groups Pn2 ${ }_{1}$ a and Pnma.

From the Rietveld analysis, it was found that the fitting was similar for both the orthorhombic systems, which agrees with the study by Khomchenko et al. [21]. However Rietveld
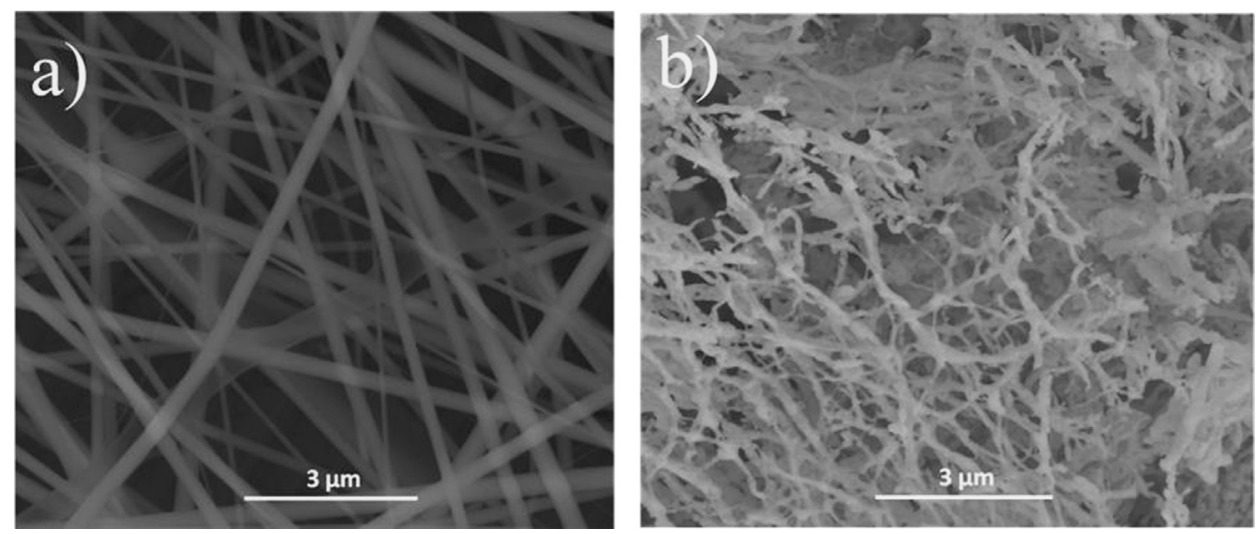

Fig. 1. SEM micrographs of (a) as spun composite nanofibers (b) phase pure BFO nanofibers obtained post annealing. 

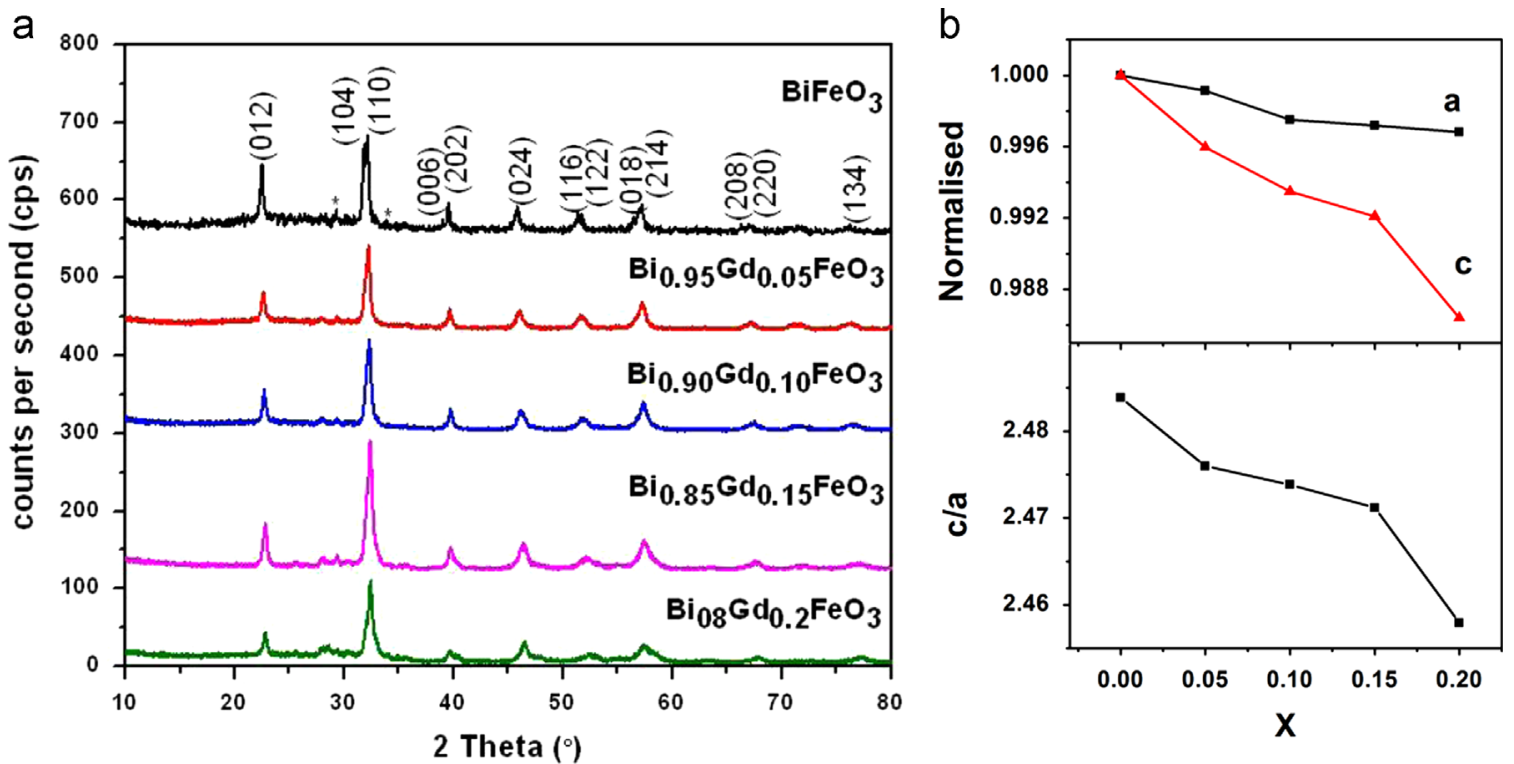

Fig. 2. (a) XRD patterns of $\mathrm{Bi}_{1-x} \mathrm{Gd}_{x} \mathrm{FeO}_{3}$ nanofibers (b) variation in unit cell parameters as a function of $\mathrm{Gd}$ concentration.

analysis shows that at $x=0.15$, the sample contained both rhombohedral and orthorhombic phases in equal proportions, and at $x=0.20$, the rhombohedral and the orthorhombic phases are in the ratio of $0.33: 1.0$. From Fig. $2 b$, we observe that both ' $a$ ' and ' $c$ ' parameters of Gd doped BFO nanofibers decrease as the amount of $\mathrm{Gd}$ dopant $(x)$ increases. The contraction of the unit cell is more pronounced along the ' $c$ ' direction than in ' $a$ ' direction. This can be attributed to contraction of the lattice with an increase in the amount of Gadolinium introduced into the system. The $\mathrm{Gd}^{3+}$ ion being smaller than the $\mathrm{Bi}^{3+}$ ion causes a contraction of the unit cell.

\subsection{Magnetic properties}

The Gd doped BFO nanofiber samples were saturated with an external magnetic field of 15,000 Gauss. The weak ferromagnetic nature of the BFO nanofibers is confirmed by $\mathrm{M}-\mathrm{H}$ hysteresis curve. The BFO nanofibers showed a magnetic saturation value of $1.95 \mathrm{emu} / \mathrm{g}$. Bulk BFO exhibits G-type antiferromagnetism with a spiral spin ordering having a wavelength of approximately $62 \mathrm{~nm}$. The restriction of the Bismuth ferrite crystallite size below $60 \mathrm{~nm}$ results in the breaking of the spiral spin ordering, giving rise to a weak ferromagnetism. This makes it more favorable for commercial applications when compared to antiferromagnetic bulk BFO. The VSM data of the pure BFO nanofibers reveal that the crystallite size is definitely below $60 \mathrm{~nm}$.

The Gd doped BFO nanofibers exhibit progressively larger saturation magnetization values with increase in amounts of Gd dopant. The $\mathrm{M}-\mathrm{H}$ hysteresis loops (Fig.3) showed a $M s$ value of $1.95 \mathrm{emu} / \mathrm{g}$ for pure BFO nanofibers. Gd doped BFO exhibit $2.4 \mathrm{emu} / \mathrm{g}, 2.5 \mathrm{emu} / \mathrm{g}, 2.96 \mathrm{emu} / \mathrm{g}$ and $4.12 \mathrm{emu} / \mathrm{g}$ as Ms values for samples with $x=0.05,0.10,0.15$ and 0.20 respectively. The large magnetic moment of the $\mathrm{Gd}$ ion explains the increase in the magnetic saturation values with corresponding increase of the $\mathrm{Gd}$ dopant. However the

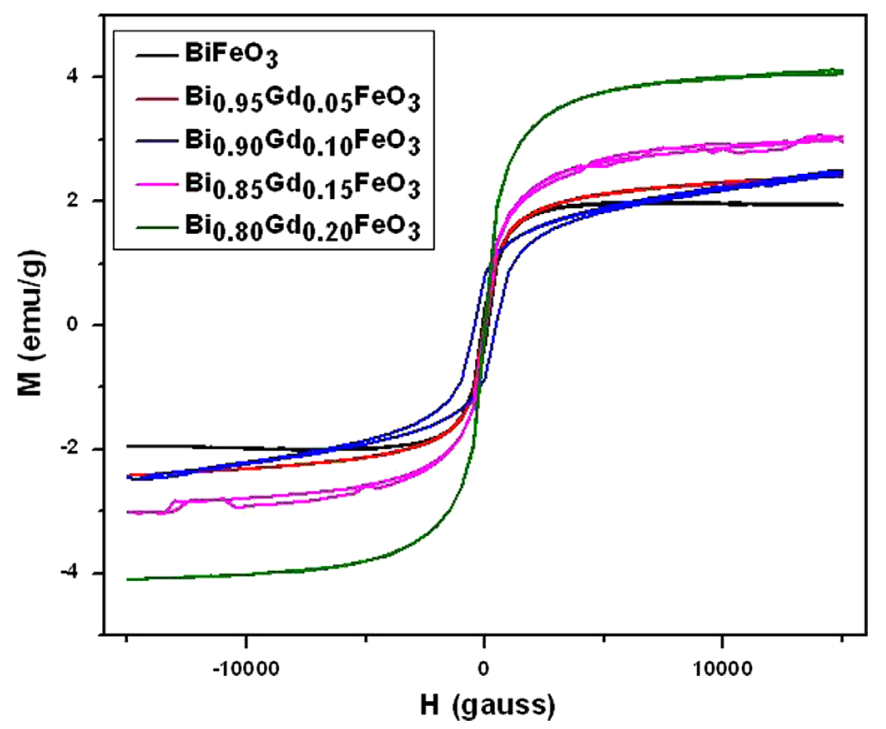

Fig. 3. $\mathrm{M}-\mathrm{H}$ hysteresis loops of $\mathrm{Bi}_{1-x} \mathrm{Gd}_{x} \mathrm{FeO}_{3}$ nanofibers.

nanofibers with higher dopant $(x=0.15,0.20)$ show instabilities in the $\mathrm{M}-\mathrm{H}$ loop which is a consequence of the presence of mixed phases as seen in the X-ray diffraction data. $\mathrm{Bi}_{0.9} \mathrm{Gd}_{0.1} \mathrm{FeO}_{3}$ nanofibers exhibit a larger coercivity of 450 Gauss as compared to coercivity of 130 Gauss of BFO nanofibers.

\subsection{Photoluminescence spectra}

The excitation spectrum of the nanofibers shows two major peaks - $363 \mathrm{~nm}$ and $401 \mathrm{~nm}$ (inset, Fig. 4 a). The intensity of the $401 \mathrm{~nm}$ is higher than the $363 \mathrm{~nm}$ peak indicating a strong absorption at the former. Thus a wavelength of $401 \mathrm{~nm}$ was chosen as the excitation wavelength to obtain the PL spectra of the nanofibers. 
a

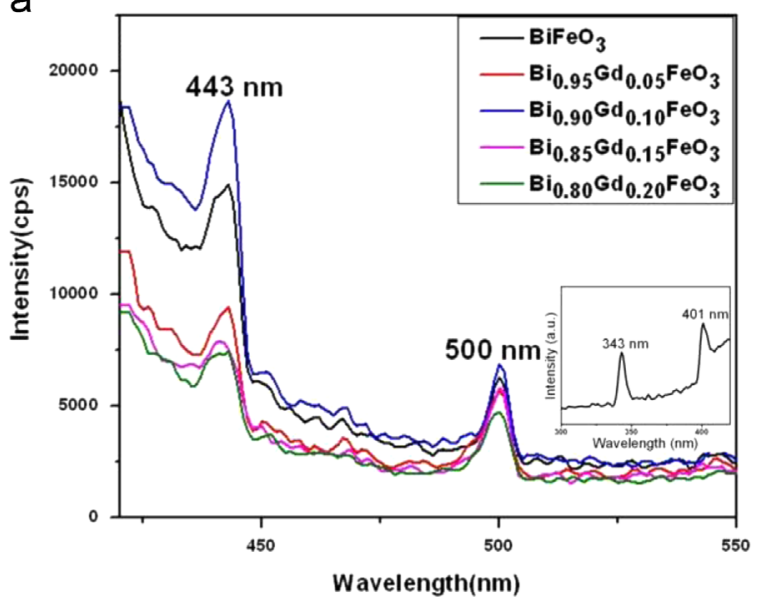

b

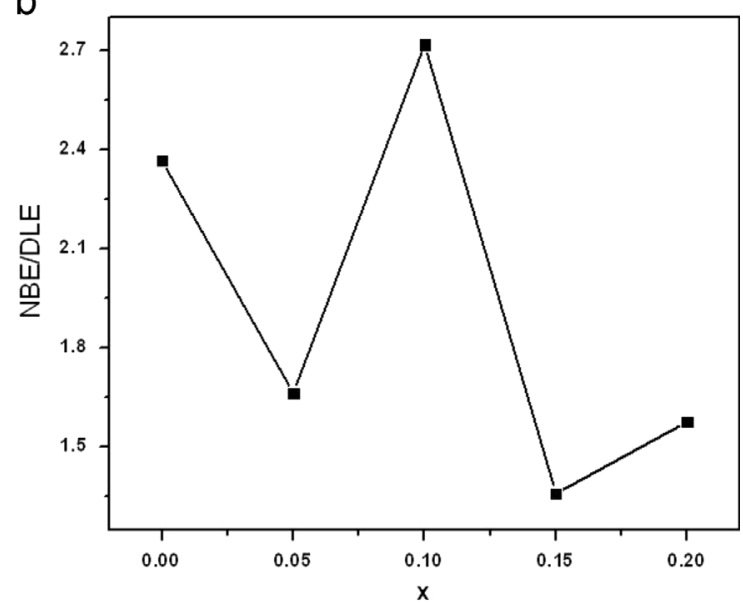

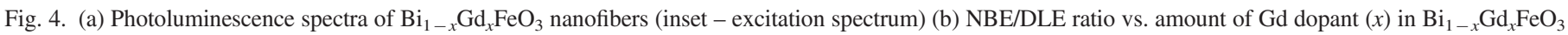
nanofibers.

The photoluminescence spectrum reveals visible light emission comprising of two major peaks - one at $443 \mathrm{~nm}(2.79 \mathrm{eV})$ and the other at $500 \mathrm{~nm}(2.48 \mathrm{eV})$. The peak at $443 \mathrm{~nm}$ corresponds to the Near Band Emission (NBE) characteristic of BFO. The other peak at $500 \mathrm{~nm}$ corresponds to the Defect Level Emission (DLE), and can be ascribed to defects such as oxygen vacancies in the crystal structure and other surface defects.

$\mathrm{Bi}_{0.9} \mathrm{Gd}_{0.1} \mathrm{FeO}_{3}$ nanofibers show maximum NBE intensity among the samples and the intensity of NBE increases without significant changes in the DLE. The intensity of NBE initially decreases for sample with $x=0.05$ from undoped BFO and then increases to a maximum when $x=0.10$ and thereafter decreases to a minimum for samples with higher Gd dopant.

In order to further understand the effect of gadolinium doping, the value of NBE/DLE ratio is plotted as a function of the concentration of Gd dopant. The highest NBE/DLE value of 2.7 is seen for $\mathrm{Bi}_{0.9} \mathrm{Gd}_{0.1} \mathrm{FeO}_{3}$ nanofibers. The high NBE/ DLE value of these nanofibers can be ascribed to the passivation of oxygen vacancies due to $\mathrm{Gd}$ doping [22,23]. This is in accordance with the studies carried out by Prashanthi et al., where the NBE/DLE value of BFO nanofibers was enhanced by annealing the samples in hydrogen; which led to the passivation of oxygen vacancies [11]. The increase in the NBE intensity by Gd doping can be explained as follows. Gd doping reduces the number of oxygen vacancies, leading to a reduction in the number of photo generated electrons trapped at the surface of the BFO nanofiber. The reduction in the number of trapped electrons leads to reduction in the height of the space charge layer [11]. This leads to larger number of radiative recombinations, thereby giving rise to a higher NBE for $\mathrm{Bi}_{0.9} \mathrm{Gd}_{0.1} \mathrm{FeO}_{3} \quad(x=0.10)$ nanofibers. In the case of nanofibers with higher levels of $\mathrm{Gd}$ dopant $(x=0.15$ and 0.20 ), the presence of secondary phases might contribute to the lowering of the photoluminescence signal. The presence of instabilities in the $\mathrm{M}-\mathrm{H}$ loop of these nanofibers along with their X-ray diffraction data is a further proof for the formation of secondary phases in these samples.

\section{Conclusions}

This study reports the synthesis of BFO and Gd doped BFO nanofibers and their structural, magnetic and photoluminescence analyses. A structural transition from rhombhohedral to orthorhombic (R3C to Pnma) system was observed as the gadolinium doping percentage increases from $x=0.10$ to 0.20 . Nanofibers with $x=0.10$ showed higher magnetic saturation and coercivity values when compared to undoped $\mathrm{BFO}$ nanofibers. The photoluminescence spectrum of $\mathrm{Bi}_{0.9} \mathrm{Gd}_{0.1} \mathrm{FeO}_{3}$ nanofibers showed an enhanced NBE in the visible spectrum due to the passivation of oxygen vacancies. The enhanced magnetic and photoluminescence properties of these nanofibers make them potential candidates for the development of multifunctional photonic and optoelectronic devices.

\section{Acknowledgments}

We would like to thank Sophisticated Analytical Instrumentation facility (SAIF), IIT Madras and Pondicherry University for providing the facilities for VSM and PL characterization respectively.

\section{References}

[1] J. Wang, J.B. Neaton, H. Zheng, V. Nagarajan, S.B. Ogale, B. Liu, et al., Epitaxial $\mathrm{BiFeO}_{3}$ multiferroic thin film heterostructures, Science 299 (2003) 1719-1722.

[2] M. Gajek, M. Bibes, S. Fusil, K. Bouzehouane, J. Fontcuberta, A. Barthelemy, et al., Tunnel junctions with multiferroic barriers, Nat. Mater. 6 (2007) 296-302.

[3] H. Bea, M. Gajek, M. Bibes, A. Barthelemy, Spintronics with multiferroics, J. Phys. Condens. Mater. 20 (2008) 434231.

[4] K. Takahashi, N. Kida, M. Tonouchi, Terahertz radiation by an ultrafast spontaneous polarization modulation of multiferroic $\mathrm{BiFeO}_{3}$ thin films, Phys. Rev. Lett. 96 (2006) 117402.

[5] S.J. Clark, Robertson, band gap and Schottky barrier heights of multiferroic $\mathrm{BiFeO}_{3}$, J. Appl. Phys. Lett. 90 (2007) 132903. 
[6] J.F. Ihlefeld, N.J. Podraza, Z.K. Liu, R.C. Rai, X. Xu, T. Heeg, et al., Optical bandgap of $\mathrm{BiFeO}_{3}$ grown by molecular - beam epitaxy, Appl. Phys. Lett. 92 (2008) 142908.

[7] S.R. Basu, L.W. Martin, Y.H. Chu, M. Gajek, R. Ramesh, R.C. Rai, et al., Photoconductivity in $\mathrm{BiFeO}_{3}$ thin films, Appl. Phys. Lett. 92 (2008) 091905.

[8] T. Choi, S. Lee, Y.J. Choi, V. Kiryukhin, S.W. Cheong, Switchable ferroelectric diode and photovoltaic effect in $\mathrm{BiFeO}_{3}$, Science 324 (2009) 63-66.

[9] R. Guo, L. You, L. Chen, D. Wu, J. Wang, Photovoltaic property of $\mathrm{BiFeO}_{3}$ thin films with 109 domains, Appl. Phys. Lett. 99 (2011) 122902.

[10] K. Prashanthi, M. Gupta, Y.Y. Tsui, T. Thundat, Effect of annealing temperature on microstructural and photoluminescence characteristics of multiferroic $\mathrm{BiFeO}_{3}$ thin films prepared by pulsed laser deposition technique, Appl. Phys. A 110 (2013) 903-907.

[11] K. Prashanthi, G. Thakur, T. Thundat, Surface enhanced strong visible photoluminescence from one dimensional multiferroic $\mathrm{BiFeO}_{3}$ nanostructures, Surf. Sci. 606 (2012) L83-L86.

[12] N. Miriyala, K. Prashanthi, T. Thundat, Oxygen vacancy dominant strong visible photoluminescence from $\mathrm{BiFeO}_{3}$ nanotubes, Phys. Status Solidi RRL 7 (2013) 668-671.

[13] A. Anshul, A. Kumar, B.K. Gupta, R.K. Kotnala, J.F. Scott, et al., Photoluminescence and time-resolved spectroscopy in multiferroic $\mathrm{BiFeO}_{3}$ : effects of electric fields and sample aging, Appl. Phys. Lett. 102 (2013) 222901.

[14] S. Goswami, D. Bhattacharya, P. Choudhury, B. Ouladdiaf, T. Chatterj, Multiferroic coupling in nanoscale $\mathrm{BiFeO}_{3}$, Appl. Phys. Lett. 99 (2011) 073106.
[15] N. Bhardwaj, S.C. Kundu, Electrospinning: a fascinating fiber fabrication technique, Biotechnol. Adv. 28 (2010) 325-347.

[16] A. Greiner, J.H. Wendorff, Electrospinning: a fascinating method for the preparation of ultrathin fibers, Angew. Chem. Int. Ed., 46, , 2007, p. 5670 .

[17] S. Anitha, D.J. Thiruvadigal, T.S. Natarajan, A study on defect controlled morphology of organic/inorganic composite nanofibers with different heat flow rates, Mater. Lett. 65 (2011) 167-170.

[18] S. Anitha, D.J. Thiruvadigal, T.S. Natarajan, In-situ preparation of high optical quality $\mathrm{ZnO}$ nanoparticles in nanofibrous PVA matrix, Mater. Lett. 65 (2011) 2872-2876.

[19] D.K. Mishra, X. Qi, Energy levels and photoluminescence properties of nickel-doped bismuth ferrite, J. Alloy. Compd. 504 (2010) 27-31.

[20] Y.H. Wang, X. Qi, The effects of nickel substitution on bismuth ferrite, Procedia Eng. 36 (2012) 455-461.

[21] V.A. Khomchenko, D.A. Kiselev, I.K. Bdikin, V.V. Shvartsman, P. Borisov, W. Kleemann, et al., Crystal structure and multiferroic properties of Gd substituted $\mathrm{BiFeO}_{3}$, Appl. Phys. Lett. 93 (2008) 262905.

[22] G.S. Lotey, N.K. Verma, Structural, magnetic, and electrical properties of Gd-doped $\mathrm{BiFeO}_{3}$ nanoparticles with reduced particle size, J. Nanopart. Res. 14 (2012) 742.

[23] J.W. Kim, S.S. Kim, H.J. Kim, W.J. Kim, C.M. Raghavan, D. Do, et al., Enhancement of ferroelectricity in gadolinium (Gd) and transition metal (Ni, Co, Cr) Co-doped $\mathrm{BiFeO}_{3}$ thin films via a chemical solution deposition technique, J. Electroceram. 30 (2013) 13-18. 